\title{
Evaluation of Rotation and Visual Outcomes After Implantation of Monofocal and Multifocal Toric Intraocular Lenses
}

\author{
Nuria Garzón, OD, MSc; Francisco Poyales, MD; Begoña Ortíz de Zárate, MD; \\ Jose Luis Ruiz-García, OD, MSc; Juan Antonio Quiroga, PhD
}

\section{ABSTRACT}

PURPOSE: To evaluate rotational stability and its influence on postoperative visual acuity of different monofocal and multifocal toric intraocular lenses (IOLs).

METHODS: A prospective interventional study was designed. Ninety-one patients with a mean age of 71.65 \pm 11.82 years were implanted with toric IOLs after phacoemulsification. Three monofocal toric IOLs (the Lentis LT [Oculentis, Berlin, Germany], enVista [Bausch \& Lomb, Rochester, NY], and AcrySof IQ [Alcon Laboratories, Inc., Fort Worth, TX]) and one multifocal toric IOL (AcrySof IQ ReSTOR; Alcon Laboratories, Inc.) were implanted. Preoperative and postoperative images were taken to calculate the misalignment due to the marking method. To evaluate rotation in the different follow-up visits, another photograph was taken 1 hour and 1, 7 and 30 days postoperatively. Refraction, uncorrected distance visual acuity (UDVA), and corrected distance visual acuity were measured 30 days postoperatively.

RESULTS: Postoperative UDVA was 0.1 logMAR or better in $64.6 \%$ of eyes implanted with monofocal IOLs and $46.4 \%$ of eyes implanted with multifocal IOLs. The enVista toric IOL showed the best UDVA compared to the other monofocal IOLs, with $81 \%$ of eyes with 0.1 logMAR or better. The mean misalignment in the total group studied was $0.07^{\circ} \pm 0.60^{\circ} ; 69.6 \%$ of monofocal IOLs and $67.9 \%$ of multifocal IOLs showed less than $5^{\circ}$ of rotation. A correlation was found between postoperative UDVA and rotation in the monofocal and multifocal IOLs implanted $(r=0.439[P<.011]$ and $=0.787[P$ $=.001]$, respectively).

CONCLUSIONS: At 1 month postoperatively, UDVA was slightly more affected by IOL rotation in multifocal than monofocal toric IOLs. The marking method was also effective.

[J Refract Surg. 2015;31(2):XX-XX.]

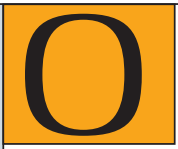

ne of the main challenges in astigmatism-correcting IOLs is ensuring precise alignment on the patient's axis of corneal astigmatism and rotational stability after IOL implantation. ${ }^{1,2}$ The slightest variation in alignment will mean that the patient will develop a postoperative astigmatism on a different axis to that of the preoperative astigmatism. ${ }^{3}$ To determine the optimal position for accurate IOL alignment, several methods have been proposed in which ink marks are placed on the corneal limbus. ${ }^{4-9}$ In some of these systems, corneal marks are placed under monocular vision (occluding one eye) to avoid the convergence produced when the patient fixates on a near target, or most often under the slit lamp with no fixed viewing position. However, none of these methods consider the possibility of cyclotorsion in monocular viewing conditions as described in previous studies. ${ }^{9,10}$ On the other hand, if the marks are made binocularly, the convergence produced when the patient views a near object is not corrected.

Regarding rotational stability, there are several studies on monofocal toric IOLs with follow-up between 1 and 6 months, where the lens position remained stable from 1 month onward. ${ }^{1,2,11-13}$ Both misalignment (differences between preoperatively calculated IOL position and real IOL position just after surgery) and rotation (differences between real IOL position just after surgery and IOL position at postoperative follow-up visits) are critical to a successful surgery in terms of visual quality. If the IOL rotates $15^{\circ}$ off axis after surgery, there will only be half correction of the power and the resultant cylinder will be shifted to a new axis. ${ }^{3}$ If a multifocal toric IOL was implanted, the rotation, misalignment, or both will probably worsen uncorrected distance visual acuity (UDVA) compared to a monofocal toric IOL; however, there

From Instituto de Oftalmología Avanzada, Madrid, Spain (NG, FP, BOD); and Universidad Complutense de Madrid, Madrid, Spain (JLR-G, JAQ).

Submitted: November 3, 2014; Accepted: December 16, 2014; Posted online: March 12, 2015

The authors have no financial or proprietary interest in the materials presented herein.

Correspondence: Nuria Garzón, OD, MSc, Instituto de Oftalmología Avanzada, c/ Galileo 104, Madrid, 28003 Spain. E-mail: ngarzon@oftalmologia-avanzada.com 
are few studies on multifocal toric IOLs and their rotational effects. ${ }^{14-16}$

To the best of our knowledge, no previous studies have been conducted to compare rotational stability and its effect on postoperative residual astigmatism between multifocal and monofocal toric IOLs. Therefore, the main objective of this study was to evaluate if there are differences in rotational stability between different monofocal and multifocal toric IOL platforms and its influence on uncorrected postoperative visual acuity. The second objective was to evaluate the effectiveness of a new marking method for reducing misalignment.

\section{PATIENTS AND METHODS}

A prospective interventional study was conducted including 91 patients (91 eyes) with a mean age of $71.65 \pm 11.82$ years (range: 27 to 95 years). Patients were implanted with toric IOLs in one or both eyes at the Instituto de Oftalmología Avanzada of Madrid during 2013. If a patient had a cataract in both eyes and if each lens calculation indicated the need for a toric lens, then only one eye from that patient was included in the study. Each eye was randomly selected. If a patient had a cataract in both eyes but only one eye needed a toric lens, that eye was included in the study. If a patient required a toric lens in only one eye because the other had no cataract or was pseudophakic, that eye was included in the study. In all cases, the patients recruited met all inclusion requirements.

All patients participated voluntarily in the study and were free to withdraw at any time. Informed consent was signed by all patients after the nature of the study and risks of participation were explained. The study was conducted in accordance with the tenets of the Declaration of Helsinki ${ }^{17}$ and was approved by the Ethics Committee of the Instituto de Oftalmología Avanzada of Madrid. Detailed demographic characteristics of the study population are shown in Table 1.

The inclusion criteria to participate were: an expected postoperative corrected distance visual acuity (CDVA) of 0.3 logMAR or better, anterior corneal astigmatism of $1.00 \mathrm{D}$ or greater, and total astigmatism (anterior and posterior corneal astigmatism) of $0.50 \mathrm{D}$ or less, with respect to the anterior astigmatism. The patients were required to have no corneal irregularities when examined under slit lamp or when examined topographically, and to show no observable macular alterations following optical coherence tomography. No patient was included who showed corneal or macular abnormalities, who required a follow-up different to that outlined in the protocol used at the center (either before or after surgery), or who required a suture.
Prior to surgery, topography with the OPD II Scan (Nidek, Hiroishi, Japan), Pentacam (Oculus Optikgeräte $\mathrm{GmbH}$, Wetzlar, Germany), laser interference biometry IOLMaster (Carl Zeiss Meditec, Jena, Germany), optical coherence tomography with the Cirrus platform (Carl Zeiss Medictec), slit-lamp examination, refraction, and CDVA were performed. Postoperative visits were scheduled at 1 day, 1 week, and 1 month after surgery. Topography, slit-lamp examination, refraction, and UDVA and CDVA were performed 1 month postoperatively.

We used the Alpins method for calculating astigmatism. The Alpins method uses three fundamental vectors: target-induced astigmatism, surgically induced astigmatism, and the difference vector. The targetinduced astigmatism is the astigmatic change (by magnitude and axis) that the surgery is intended to induce and the surgically induced astigmatism is the amount and axis of astigmatic change the surgery actually induces. The difference vector is the induced astigmatic change (by magnitude and axis) that would enable the initial surgery to achieve the intended target.

\section{IOLS}

Three monofocal toric IOLs (the Lentis LT [LU-313T; Oculentis, Berlin, Germany], enVista [Bausch \& Lomb, Rochester, NY], and AcrySof IQ toric IOL [SN6A T3T9; Alcon Laboratories, Inc., Fort Worth, TX]) and one multifocal toric IOL (the AcrySof IQ ReSTOR [SND1 T1-T5; Alcon Laboratories, Inc.) were implanted. More technical details are shown in Table A (available in the online version of this article).

\section{MARKING MethOd}

During the preoperative preparation of the patient, two marks were placed at the limbus using a fine-tipped gentian violet marker (Devon Skin Marker, Fine Tip 151; Covidien, Dublin, Ireland) on the horizontal axis $\left(0^{\circ}\right.$ to $\left.180^{\circ}\right)$ at the 9- and 3-o'clock positions under slitlamp observation (Figure 1A). A photograph was taken of the patient standing $2.5 \mathrm{~m}$ or more from the camera, with the head in normal position without the use of a chinrest or other support. Both eyes were open, thus avoiding the cyclotorsion that can occur if one is closed. Images were taken at a distance that avoided cyclotorsion when looking at a close object. The position of the marks made by the surgeon were measured on the image acquired. Thus, once a patient was supine, the exact position of the mark (made with violet blue) was known, independent of any cyclotorsion that might take place.

The camera used was a Coolpix P90 (Nikon, Tokyo, Japan), with a 26.0 - to $624.0-\mathrm{mm}$ lens (at $35 \mathrm{~mm}$ ), a charge-coupled device of 12.10 effective megapixels, 
TABLE 1

Preoperative Patient Demographics

\begin{tabular}{|c|c|c|c|c|c|}
\hline \multirow[b]{2}{*}{ Parameter } & \multicolumn{5}{|c|}{ Mean \pm SD (Range) } \\
\hline & Total & $\begin{array}{l}\text { AcrySof IQ } \\
\text { Toric IOL }\end{array}$ & enVista Toric IOL & Lentis LT IOL & $\begin{array}{c}\text { AcrySof IQ } \\
\text { ReSTOR Toric IOL }\end{array}$ \\
\hline Mean age (y) & $\begin{array}{c}71.65 \pm 11.82 \\
(27 \text { to } 95)\end{array}$ & $\begin{array}{l}77.21 \pm 8.60 \\
\quad(58 \text { to } 95)\end{array}$ & $\begin{array}{c}71.19 \pm 9.34 \\
(57 \text { to } 89)\end{array}$ & $\begin{array}{l}72.07 \pm 11.41 \\
\quad(57 \text { to } 93)\end{array}$ & $\begin{array}{c}64.25 \pm 14.72 \\
\quad(27 \text { to } 91)\end{array}$ \\
\hline Gender (female/male) & $52 / 39$ & $16 / 12$ & $10 / 11$ & $10 / 4$ & $16 / 12$ \\
\hline Mean corneal astigmatism (D) & $\begin{array}{l}1.87 \pm 0.67 \\
(1.00 \text { to } 4.55)\end{array}$ & $\begin{array}{l}1.72 \pm 0.85 \\
(1.00 \text { to } 4.55)\end{array}$ & $\begin{array}{l}1.89 \pm 0.57 \\
(1.01 \text { to } 3.31)\end{array}$ & $\begin{array}{l}1.93 \pm 0.66 \\
(1.07 \text { to } 3.27)\end{array}$ & $\begin{array}{l}1.91 \pm 0.61 \\
(1.00 \text { to } 3.08)\end{array}$ \\
\hline \multicolumn{6}{|l|}{ Mean keratometry (D) } \\
\hline Flat & $43.10 \pm 1.61$ & $43.34 \pm 1.61$ & $43.63 \pm 1.33$ & $43.13 \pm 2.23$ & $42.63 \pm 1.54$ \\
\hline Mean refractive cylinder (D) & $\begin{array}{l}2.03 \pm 0.86 \\
(0.50 \text { to } 4.25)\end{array}$ & $\begin{array}{l}2.26 \pm 0.95 \\
(0.75 \text { to } 4.25)\end{array}$ & $\begin{array}{l}2.09 \pm 0.58 \\
(1.25 \text { to } 3.50)\end{array}$ & $\begin{array}{l}1.71 \pm 0.87 \\
(0.50 \text { to } 3.50)\end{array}$ & $\begin{array}{l}1.85 \pm 0.99 \\
(0.50 \text { to } 3.75)\end{array}$ \\
\hline Preoperative CDVA (logMAR) & $\begin{array}{c}0.19 \pm 0.17 \\
(0.01 \text { to } 0.82)\end{array}$ & $\begin{array}{c}0.30 \pm 0.21 \\
(0.05 \text { to } 0.52)\end{array}$ & $\begin{array}{c}0.15 \pm 0.17 \\
(0 \text { to } 0.70)\end{array}$ & $\begin{array}{l}0.22 \pm 0.13 \\
(0 \text { to } 0.82)\end{array}$ & $\begin{array}{c}0.10 \pm 0.12 \\
(0 \text { to } 0.52)\end{array}$ \\
\hline IOL power (sphere) (D) & $20.79 \pm 5.64$ & $19.98 \pm 5.98$ & $20.47 \pm 4.33$ & $20.67 \pm 3.78$ & $19.72 \pm 6.82$ \\
\hline IOL power (cylinder) (D) & $1.91 \pm 1.22$ & $2.43 \pm 0.97$ & $2.32 \pm 0.55$ & $2.84 \pm 0.52$ & $2.38 \pm 0.72$ \\
\hline
\end{tabular}

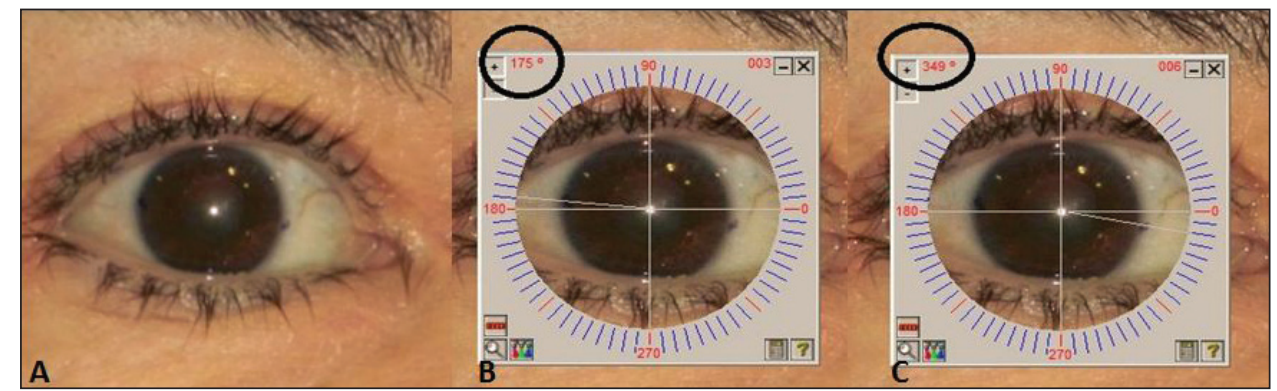

Figure 1. (A) Two marks placed at the limbus on the horizontal axis $\left(0^{\circ}\right.$ to $\left.180^{\circ}\right)$ at the 9- and 3-o'clock positions performed under slit-lamp observation. ( $B$ and $C$ ) The exact position of the marks and their angular difference with respect to the "real" $0^{\circ}$ to $180^{\circ}$ line. and a $\times 24$ (optical) $/ \times 4$ (digital) zoom. The camera was placed on a tripod and connected to a bubble level to ensure the photograph was perfectly aligned. The camera lens was positioned at eye level and at $2.5 \mathrm{~m}$ or more. The optical zooming option allowed us to simultaneously photograph both eyes. The photograph was then exported to a computer equipped with software that enables precise angle measurements by means of the Scale 2.0 package (AQ1Software R. Sgrillo, Brazil).

On the photograph, the angle the corneal marks form with the horizontal line that transects the corneal reflex was measured (Figures 1B-1C). This gives the exact position of the marks and their angular difference with respect to the "real" $0^{\circ}$ to $180^{\circ}$ line. With the patient lying down, using the Méndez ring and taking reference of the previous marks, the axis was to be aligned with the IOL's index marks, considering the angle difference observed in the photographic measurements was marked. The preoperative image and last photograph obtained during surgery showing the final axis position of the IOL were compared to calculate the misalignment due to the marking method. To assess the rotation in the different follow-up visits, another photograph was taken 1 hour and 1, 7, and 30 days after surgery.

A triangulation method was used for measuring the rotation. This required the determination of the limbal edge, the determination of the IOL edge (optical 
zone), one reproducible marker (eg, a vessel crossover, a pupil mark) on the eye, and the marks on the IOL. The IOL diameter is the same whatever the distance of the slit lamp to the eye, allowing the distance measured to be harmonized between one photograph and another. The triangle is formed by the IOL center, IOL mark, and biometric marker (eg, a vessel crossover). The change in triangle rotation determines the IOL rotation. Clockwise rotation was counted as positive rotation and anti-clockwise rotation as negative rotation.

\section{Cataract Surgery Procedure}

All surgeries were performed by the same experienced surgeon. The cylinder power and axis were calculated using the software provided by the manufacturers and taking into account the keratometric readings measured with a topographer. Phacoemulsification was performed through a $2.2-\mathrm{mm}$ clear corneal incision. The astigmatism induced by the surgeon (FP) was previously known. The lenses were introduced via a 2.2-mm incision and the surgeon induced an astigmatism of $0.1 \mathrm{D}$ in the right eye (temporal incision). In the left eye, the superior incision produced an astigmatism of $0.2 \mathrm{D}$. This was taken into account when the power of the lenses to be implanted was calculated.

In any case, it was necessary to open the incision for inserting the foldable IOL with the injector. To shield the corneal endothelial cells, a dispersive viscoelastic (Viscoat; Alcon Laboratories, Inc.) was first injected into the anterior chamber followed by another cohesive viscoelastic (Amvisc Plus; Bausch \& Lomb) injected below the first one. The incision was not sutured and the eyes were not patched.

\section{Statistical ANALYSIS}

Data were analyzed by SPSS version 17.0 (SPSS, Inc., Chicago, IL) for Windows. The values presented are the mean \pm standard deviation of the values obtained. Normality of distribution was assessed using the Kolmogorov-Smirnov test. One-way repeated measure analysis of variance was used to assess the trend of IOL rotation during postoperative follow-up visits. Student's $t$ test for related samples was used to compare preoperatively calculated IOL position with IOL position just after surgery (misalignment). To correlate rotation (absolute values) at 30 days and misalignment values with UDVA and astigmatism postoperative power, Pearson bivariate regression was used. A $P$ value of less than .05 was considered statistically significant.

\section{RESULTS}

Detailed demographic characteristics of the study population are shown in Table 1. All postoperative re- sults regarding visual acuity, refraction, and rotation are shown in Table 2.

Postoperative UDVA was 0.1 logMAR (20/25 Snellen) or better in $64.6 \%$ of eyes implanted with monofocal toric IOLs and $46.4 \%$ of eyes implanted with multifocal toric IOLs (Figure A, available in the online version of this article). The enVista toric IOL showed the best UDVA compared to the other monofocal toric IOLs, with $81 \%$ of eyes with 0.1 logMAR (20/25 Snellen) or better (Figure 2).

Mean CDVA showed a statistically significant increase after IOL implantation in all lenses studied. The mean preoperative CDVA was $0.19 \pm 0.17$ (range: 0.01 to 0.82 ) and mean postoperative CDVA was $0.02 \pm 0.05$ (range: 0.00 to 0.30$)(P<.05$; Student's $t$ test).

The mean spherical equivalent for all patients decreased after surgery to $-0.10 \pm 0.51 \mathrm{D}$ (range: -1.25 to $2.38 \mathrm{D})$ and was statistically significant compared to mean preoperative spherical equivalent $(P<.05$; Student's $t$ test), but no differences were found with the mean targeted spherical equivalent, which was - 0.03 $\pm 0.40 \mathrm{D}$ (range: -1.95 to $0.75 \mathrm{D}$ ). The mean difference between targeted spherical equivalent and mean postoperative spherical equivalent was $-0.07 \pm 0.46 \mathrm{D}$, and no significant differences were seen between the postoperative spherical equivalent and the targeted value programmed after performing the lens calculation. No statistical differences were found between the IOLs implanted, the enVista being the lens with the highest mean spherical equivalent and the AcrySof IQ ReSTOR toric IOL with the lowest mean spherical equivalent after surgery.

Mean cylinder decreased in all patients from 2.03 $\pm 0.86 \mathrm{D}$ to $0.61 \pm 0.57 \mathrm{D}$ after surgery. Statistical differences were found for all lenses studied $(P<.05$; Student's $t$ test). Postoperative cylinder between plano and $0.50 \mathrm{D}$ was found in $73.3 \%$ and $43.9 \%$ of eyes with monofocal and multifocal IOLs, respectively (Figure B). The enVista IOL showed more eyes with postoperative cylinder between plano and $0.50 \mathrm{D}$ than the other monofocal toric IOLs studied, with more than $80 \%$ of eyes within this range (Figure 3).

A new marking method was also evaluated in this study. The misalignment was the difference between preoperatively and postoperatively calculated IOL positions. The mean misalignment in the total group studied was $0.07^{\circ} \pm 0.60^{\circ}$ (range: $-2.00^{\circ}$ to $3.00^{\circ}$ ). Table 2 shows misalignment values for the different IOLs. No statistical difference was found between preoperatively and postoperatively calculated IOL positions for any IOL studied $(P<.05$; Student's $t$ test).

Rotation of the lenses was measured 1 hour and 1, 7, and 30 days after surgery. Figure $\mathbf{C}$ (available in the on- 
TABLE 2

\section{Postoperative Results of Visual Acuity to Refraction to Misalignment and Rotation With the Different IOLs Implanted}

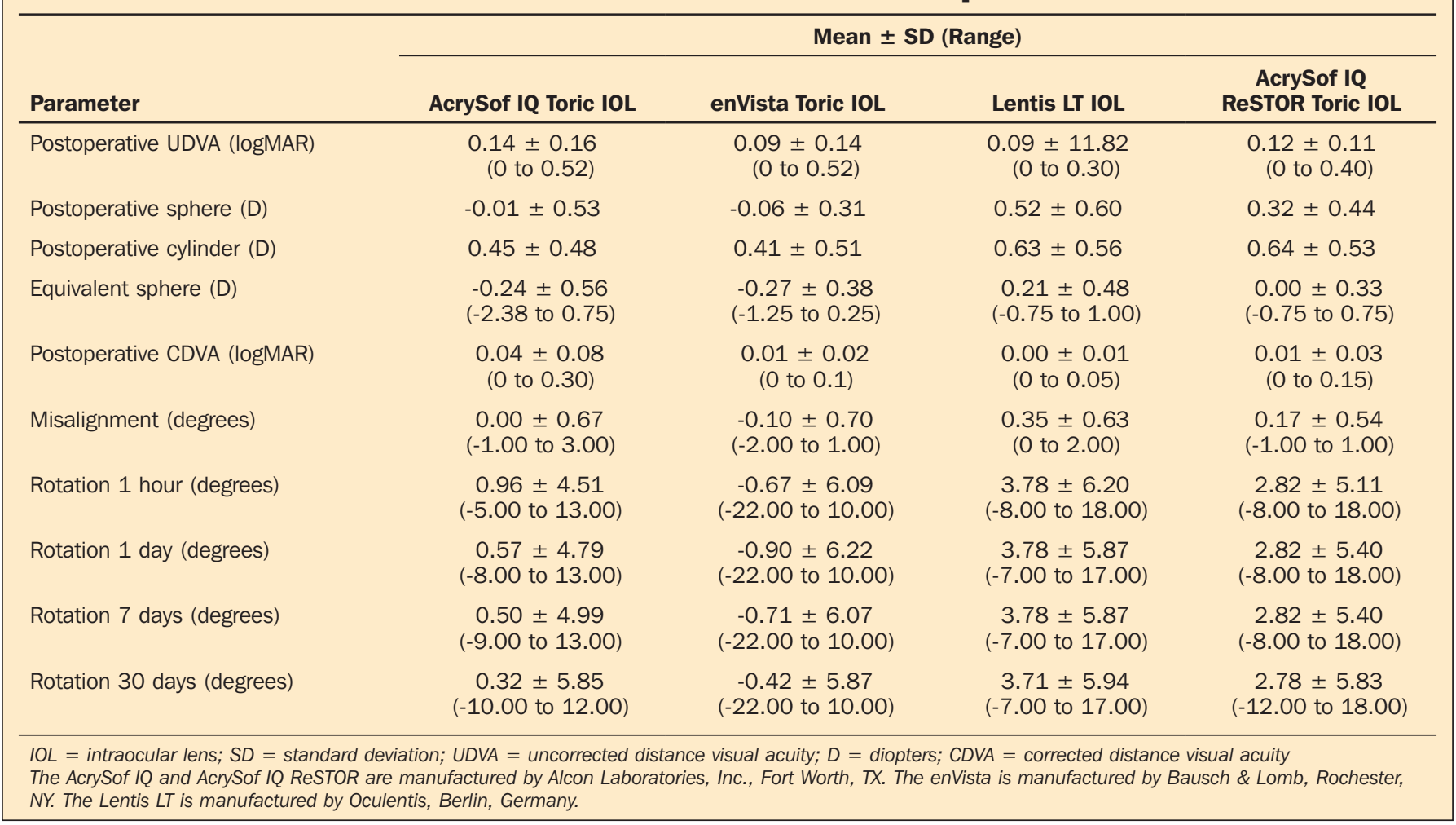

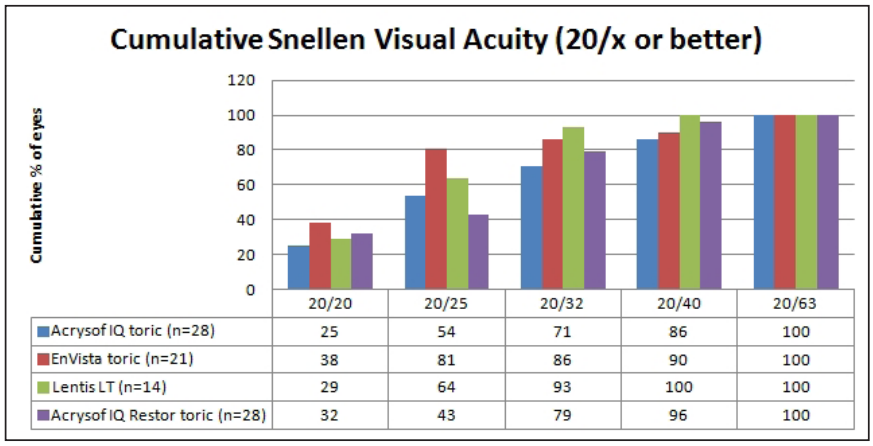

Figure 2. Comparison of postoperative uncorrected distance visual acuity frequency at 30 days between different monofocal toric intraocular lenses (the AcrySof IQ [Alcon Laboratories, Inc., Fort Worth, TX], enVista [Bausch \& Lomb, Rochester, NY] and Lentis LT [Oculentis, Berlin, Germany]) for different visual acuity values.

line version of this article) shows rotation values of the monofocal and multifocal IOL implanted at the different follow-up visits. No statistical differences between visits were found between monofocal and multifocal IOLs $(P>.05$; one-way repeated measure analysis of variance).

One hour after surgery, the lens that showed the most rotation was that with the plate design (Lentis

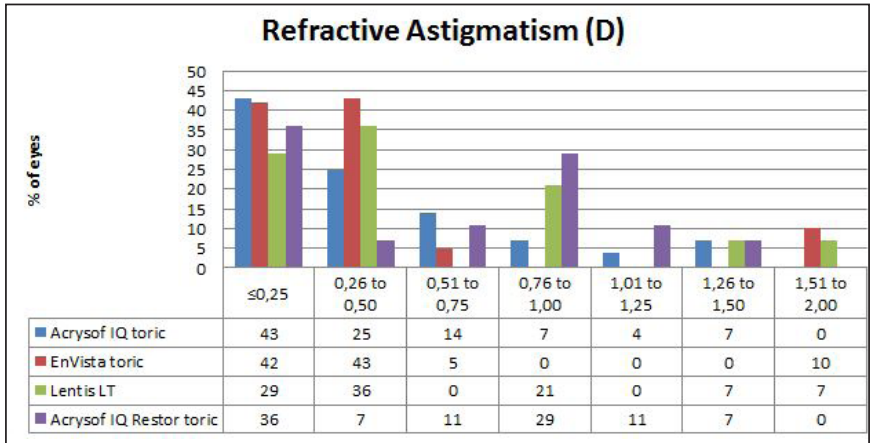

Figure 3. Comparison of postoperative refractive astigmatism frequency at 30 days between different monofocal toric intraocular lenses (the AcrySof IQ [Alcon Laboratories, Inc., Fort Worth, TX], enVista [Bausch \& Lomb, Rochester, NY] and Lentis LT [Oculentis, Berlin, Germany]) for different powers.

LT). The enVista lens was the most stable and the only one to show anti-clockwise rotation; all of the others showed clockwise rotation (Figure 4).

At 30 days postoperatively, $69.6 \%$ of monofocal IOLs and $67.9 \%$ of multifocal IOLs showed less than $5^{\circ}$ of rotation. Fifteen $(18.9 \%)$ eyes implanted with monofocal IOLs and $5(17.8 \%)$ eyes implanted with multifocal IOLs showed more than $10^{\circ}$ of rotation. 


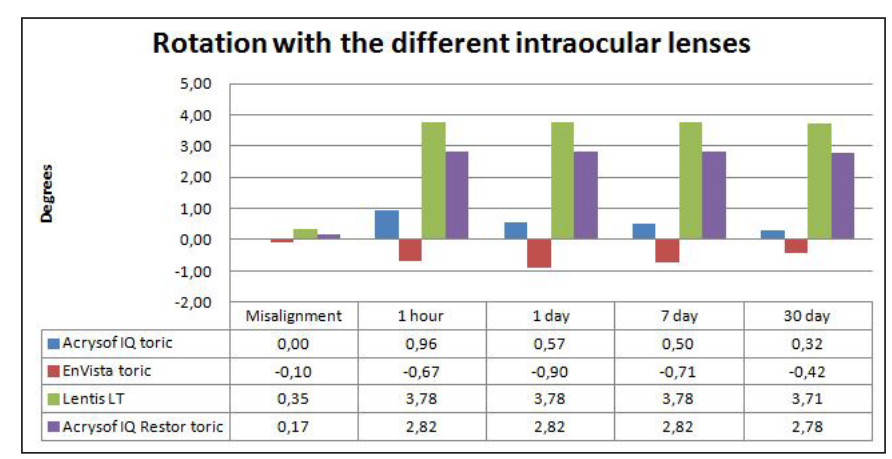

Figure 4. Rotation observed for each design. Negative values indicate an anti-clockwise rotation and positive values indicate a clockwise rotation. The AcrySof IQ and AcrySof IQ ReSTOR are manufactured by Alcon Laboratories, Inc., Fort Worth, TX. The enVista is manufactured by Bausch \& Lomb, Rochester, NY. The Lentis LT is manufactured by Oculentis, Berlin, Germany.

At 30 days postoperatively, $90.5 \%$ of eyes implanted with the enVista toric IOL showed less than $5^{\circ}$ of rotation, whereas the figures for the AcrySof IQ toric IOL and Lentis LT IOL were $64.3 \%$ and $64.6 \%$, respectively. More than $10^{\circ}$ of rotation was observed in $21.4 \%$ of eyes with the AcrySof toric IOL, $9.5 \%$ with the enVista toric IOL, and $14.28 \%$ with the Lentis LT IOL.

There was a correlation between postoperative UDVA and postoperative cylinder power in the monofocal and multifocal IOLs implanted $(r=0.807, P<$ .001 and $r=0.631, P<.001$, respectively), between postoperative UDVA and rotation 30 days after the surgery in multifocal IOLs ( $r=0.787, P=.001)$, and between postoperative UDVA and rotation 30 days after surgery in eyes implanted with monofocal IOLs $(r=$ 0.439, $P=.011)$.

\section{DISCUSSION}

In this study, a new toric IOL marking method was proposed that minimizes patient movement errors by having the patient sitting or standing while adopting a normal head position. The marks were made at a distance at which there should be no convergence in bilateral viewing conditions, avoiding possible monocular cyclotorsion. The main error of all available methods published to date was regarding the marking because the general idea was that those marks performed in the $0^{\circ}$ to $180^{\circ}$ axis corresponded just to that meridian. It is almost impossible to avoid the typical patient reflex of small eye movements, eyes closing, or head turning when seeing the marker approaching. These changes can generate errors of up to $15^{\circ}$ and therefore will affect the final refraction after surgery.

The main advantage of the marking method described in the current study compared to existing ones was the checking of the real axis marked with gentian violet, thus providing accurate and precise marks of the real axis instead of relative position marks.

Seventy-five percent of patients did not present misalignment and only 3 of 91 patients had $2^{\circ}$ or more of misalignment. In the comparison of the theoretical position of the toric lens (obtained by calculation) with that observed in the last image obtained during cataract surgery, no significant difference was seen. These results demonstrated the usefulness of this marking method because it clearly reduced the misalignment after surgery.

There are several studies on toric IOL rotation, ${ }^{1,2,11-13}$ but to the best of our knowledge this is the first study to compare rotational stability between monofocal and multifocal toric IOLs and between different designs. Moreover, in this study, monofocal toric IOLs were compared with the surgery protocol performed by the same surgeon. Toric IOL rotation after implantation was due to various mechanisms. Factors that may induce toric IOL rotation include postoperative intraocular pressure fluctuations, ${ }^{18}$ reduced friction between the haptics and the capsular bags produced by an incomplete ophthalmic viscoelastic device removal, ${ }^{19}$ or capsule size ${ }^{20}$ IOL design and materials,${ }^{12}$ and compression of the IOL from capsule shrinkage. ${ }^{21}$

The current results indicated that rotation occurs in all of the lenses during the first hour after surgery and remains stable during the first month. These results were in agreement with others demonstrating that rotation occurred 1 hour after surgery. ${ }^{12}$ The fact that the rotation takes place so quickly suggests that it might be a consequence of poor friction between the haptic and the capsular bag due to an incomplete ophthalmic viscoelastic device clearance, as previously suggested. Alternatively, it could be due to the size difference between the capsular bag and IOL diameter. A large diameter of the lens with respect to the capsule would produce more friction and therefore a minimal risk of rotation, but could provoke distortion of the capsular bag and zonules. Nevertheless, it is important to note that the preoperative calculation of the capsule size is complicated and there is not a clear relation between parameters such as corneal diameter and capsule size. ${ }^{22}$

When the mean rotation among the different toric monofocal IOLs was compared, the AcrySof IQ and enVista toric lenses showed less rotation than the Lentis LT lens, although none of the lenses showed a mean rotation of more than $5^{\circ}$. Both the AcrySof IQ toric and enVista lenses are open-loop haptic lenses, whereas the Lentis LT is a plate-haptic lens. These characteristics may be the reasons for these variations in the rotation among the different lenses. Neverthe- 
less, it is important to indicate that these results were in disagreement with those described by Patel et al. ${ }^{23}$ because they described plate-haptic lenses as more stable in terms of rotation than open-loop haptic lenses. Interestingly, Prinz et al. ${ }^{12}$ found a similar rotational behavior between both lens designs. Surprisingly, the multifocal design of the AcrySof IQ toric IOL showed $2^{\circ}$ more rotation than the monofocal design. It remains unclear whether the haptic design plays an important role in lens rotation and hence the controversy about which haptic design is better for rotational stability. More studies are needed to clarify this point.

Stephenson $^{24}$ reported $9 \%$ of patients implanted with the enVista toric lens showed a rotation of greater than $5^{\circ}$. This result is similar to that achieved with this lens in the current study. Our results were slightly worse than those reported by Chua et al. ${ }^{11}$ for the AcrySof monofocal lens. The literature contained no information on the Lentis LT lenses for any comparison to be made. With respect to the AcrySof toric multifocal lens, our results contrast with those of other studies $^{14}$ that report a lens rotation of no more than $10^{\circ}$; in the current study, $17.68 \%$ of patients showed a rotation of at least this amount.

Postoperative UDVA was similar for all monofocal toric IOLs and also when we compared monofocal with multifocal lenses. The results presented here confirm the results described by other studies indicating that the use of toric IOLs permits patients to achieve good UDVA after surgery. ${ }^{15,25}$ With respect to UDVA, $0.1 \log$ MAR or better was achieved with $66.4 \%$ of the monofocal toric lenses and with $46.4 \%$ of the multifocal toric lenses. Other studies with monofocal lenses have reported similar results with the AcrySof toric IOL $^{26-28}$ and enVista toric IOL. ${ }^{24}$ The literature contains no reports on the Lentis LT lenses that would allow a comparison. Other studies ${ }^{14}$ on the same multifocal lens report similar results to those of the current study.

One of the goals of the current study was to see how IOL rotation affects UDVA when different designs are used and to compare monofocal and multifocal lenses. A correlation was found between UDVA and rotation with monofocal and multifocal lenses, which was stronger in the case of toric multifocal IOLs. The correlation was positive in both cases, indicating that the higher the lens rotation, the higher the logMAR UDVA value obtained, and therefore worse visual acuity. It was expected that patients' visual acuity would be more affected by a greater rotation of the multifocal lenses. In this regard, the distorted image caused by residual astigmatism due to rotation was added to the loss of image quality due to the apodized diffractive design of multifocal toric IOLs. Still, the UDVA achieved by patients with multifocal toric IOLs was comparable to that obtained by patients with monofocal toric IOLs.

The new marking method for the alignment of toric IOLs is effective and it is not influenced by convergence, monocular cyclotorsion, or both. On the other hand, all toric IOLs studied (both monocular and multifocal) showed rotation 1 hour after surgery. This rotation was stable during the first month and had slightly more influence on UDVA after multifocal than monofocal toric IOL implantation.

\section{AUTHOR CONTRIBUTIONS}

Study concept and design (NG, FP); data collection (NG, BOD, $J L R-G)$; analysis and interpretation of data (NG, FP, JAQ); drafting of the manuscript (BOD, JLR-G); critical revision of the manuscript (NG, FP, JAQ); statistical expertise (NG, JAQ); supervision (NG, FP)

\section{REFERENCES}

1. Bachernegg A, Ruckl T, Riha W, Grabner G, Dexl AK. Rotational stability and visual outcome after implantation of a new toric intraocular lens for the correction of corneal astigmatism during cataract surgery. J Cataract Refract Surg. 2013;39:1390-1398.

2. Wolffsohn JS, Buckhurst PJ. Objective analysis of toric intraocular lens rotation and centration. J Cataract Refract Surg. 2010;36:778-782.

3. Felipe A, Artigas JM, Diez-Ajenjo A, Garcia-Domene C, Alcocer P. Residual astigmatism produced by toric intraocular lens rotation. J Cataract Refract Surg. 2011;37:1895-1901.

4. Jain AK, Gupta R, Chakma P. A simple technique for corneal marking before toric intraocular lens implantation. J Cataract Refract Surg. 2013;39:966-967.

5. Roumeliotis GA, Hutnik CM. A simple, reproducible, and cost effective axis marking system for toric lens implantation. J Refract Surg. 2012;28:12-13.

6. Visser N, Berendschot TT, Bauer NJ, Jurich J, Kersting O, Nuijts RM. Accuracy of toric intraocular lens implantation in cataract and refractive surgery. J Cataract Refract Surg. 2011;37:1394-1402.

7. Cha D, Kang SY, Kim SH, Song JS, Kim HM. New axis-marking method for a toric intraocular lens: mapping method. J Refract Surg. 2011;27:375-379.

8. Graether JM. Simplified system of marking the cornea for a toric intraocular lens. J Cataract Refract Surg. 2009;35:1498-1500.

9. Viestenz A, Seitz B, Langenbucher A. Evaluating the eye's rotational stability during standard photography: effect on determining the axial orientation of toric intraocular lenses. J Cataract Refract Surg. 2005;31:557-561.

10. Popp N, Hirnschall N, Maedel S, Findl O. Evaluation of 4 corneal astigmatic marking methods. J Cataract Refract Surg. 2012;38:2094-2099.

11. Chua WH, Yuen LH, Chua J, Teh G, Hill WE. Matched comparison of rotational stability of 1-piece acrylic and plate-haptic silicone toric intraocular lenses in Asian eyes. J Cataract Refract Surg. 2012;38:620-624.

12. Prinz A, Neumayer T, Buehl W, et al. Rotational stability and posterior capsule opacification of a plate-haptic and an open-loop-haptic intraocular lens. J Cataract Refract Surg. 2011;37:251-257.

13. Buckhurst PJ, Wolffsohn JS, Naroo SA, Davies LN. Rotational and 
centration stability of an aspheric intraocular lens with a simulated toric design. J Cataract Refract Surg. 2010;36:1523-1528.

14. Ferreira TB, Marques EF, Rodrigues A, Montes-Mico R. Visual and optical outcomes of a diffractive multifocal toric intraocular lens. J Cataract Refract Surg. 2013;39:1029-1035.

15. Alfonso JF, Knorz M, Fernandez-Vega L, et al. Clinical outcomes after bilateral implantation of an apodized +3.0 D toric diffractive multifocal intraocular lens. J Cataract Refract Surg. 2014;40:51-59.

16. Bellucci R, Bauer NJ, Daya SM, et al. Visual acuity and refraction with a diffractive multifocal toric intraocular lens. J Cataract Refract Surg. 2013;39:1507-1518.

17. Williams JR. The Declaration of Helsinki and public health. Bull World Health Organ. 2008;86:650-652.

18. Pereira FA, Milverton EJ, Coroneo MT. Miyake-Apple study of the rotational stability of the AcrySof Toric intraocular lens after experimental eye trauma. Eye (Lond). 2010;24:376-378.

19. Chang DF. Early rotational stability of the longer Staar toric intraocular lens: fifty consecutive cases. J Cataract Refract Surg. 2003;29:935-940

20. Ohmi S. Decentration associated with asymmetric capsular shrinkage and intraocular lens size. J Cataract Refract Surg. 1993;19:640-643.

21. Ohmi S, Tanaka S, Saika S, Ohnishi Y. Silicone and poly(methyl methacrylate) lens decentration associated with asymmetric capsule shrinkage in rabbits. J Cataract Refract Surg. 1999;25:1147-1150.

22. Khng C, Osher RH. Evaluation of the relationship between corneal diameter and lens diameter. J Cataract Refract Surg. 2008;34:475-479.

23. Patel CK, Ormonde S, Rosen PH, Bron AJ. Postoperative intraocular lens rotation: a randomized comparison of plate and loop haptic implants. Ophthalmology. 1999;106:2190-2195.

24. Stephenson M. Early users share the enVista experience. Review of Ophthalmology. 2013;January(available: http:// www.reviewofophthalmology.com/content/t/intraocular_ lenses/c/38573/)

25. Visser N, Bauer NJ, Nuijts RM. Toric intraocular lenses: historical overview, patient selection, IOL calculation, surgical techniques, clinical outcomes, and complications. J Cataract Refract Surg. 2013;39:624-637.

26. Mendicute J, Irigoyen C, Aramberri J, Ondarra A, Montés-Micó R. Foldable toric intraocular lens for astigmatism correction in cataract patients. J Cataract Refract Surg. 2008;34:601-607.

27. Dardzhikova A, Shah CR, Gimbel HV. Early experience with the AcrySof toric IOL for the correction of astigmatism in cataract surgery. Can J Ophthalmol. 2009;44:269-273.

28. Tsinopoulos IT, Tsaousis KT, Tsakpinis D, Ziakas NG, Dimitrakos SA. Acrylic toric intraocular lens implantation: a single center experience concerning clinical outcomes and postoperative rotation. Clin Ophthalmol. 2010;4:137-142.

\section{AUTHOR QUERIES}

AQ1 Please provide the full manufacturer and location (inluding manufacturer name, city, and country). 


\section{TABLE A}

\section{Characteristic Details of Implanted IOLs}

\begin{tabular}{lcccc}
\hline Parameter & AcrySof IQ Toric IOL & enVista Toric IOL & Lentis LT IOL & AcrySof IQ ReSTOR Toric IOL \\
\hline Type & Toric & Toric & Toric & Multifocal toric \\
Design & Open-loop haptic & Open-loop haptic & Plate-haptic & Open-loop haptic \\
Optic size & $6.0 \mathrm{~mm}$ & $6.0 \mathrm{~mm}$ & $6.0 \mathrm{~mm}$ & $6.0 \mathrm{~mm}$ \\
Overall length & $13.0 \mathrm{~mm}$ & $12.50 \mathrm{~mm}$ & $11.0 \mathrm{~mm}$ & $13.0 \mathrm{~mm}$ \\
Optic design & Biconvex toric & Aspheric & Biconvex aspheric & Biconvex apodized \\
& aspheric & monotoric & diffractive aspheric toric \\
IOL powers (D) & +6 to +30 & +10 to +30 & +10 to +30 & +6 to +30 \\
IOL cylinders (D) & 1.50 to $6.00($ T3-T9) & 1.25 to 5.75 & 1.50 to $5.25($ T1-T6) & 1.00 to 3.00 (T2-T5) \\
Haptic angulation & $0^{\circ}$ & $0^{\circ}$ & $0^{\circ}$ & $0^{\circ}$ \\
Refractive index & 1.55 & 1.54 & 1.46 & 1.55
\end{tabular}

$I O L=$ intraocular lens; $D=$ diopters

The AcrySof IQ and AcrySof IQ ReSTOR are manufactured by Alcon Laboratories, Inc., Fort Worth, TX. The enVista is manufactured by Bausch \& Lomb, Rochester, NY. The Lentis LT is manufactured by Oculentis, Berlin, Germany.

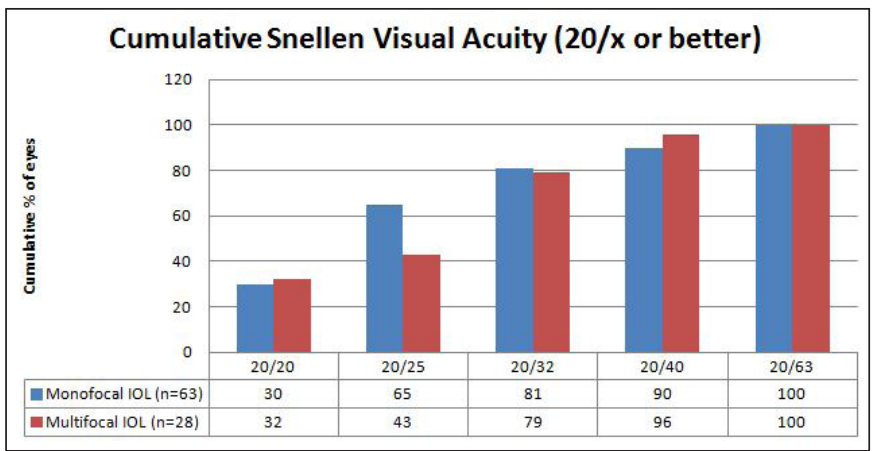

Figure A. Comparison of postoperative uncorrected distance visual acuity frequency at 30 days between monofocal toric intraocular lenses (IOLs) ( $\mathrm{n}$ $=63)$ and multifocal toric IOLs $(n=28)$ for different visual acuity values.

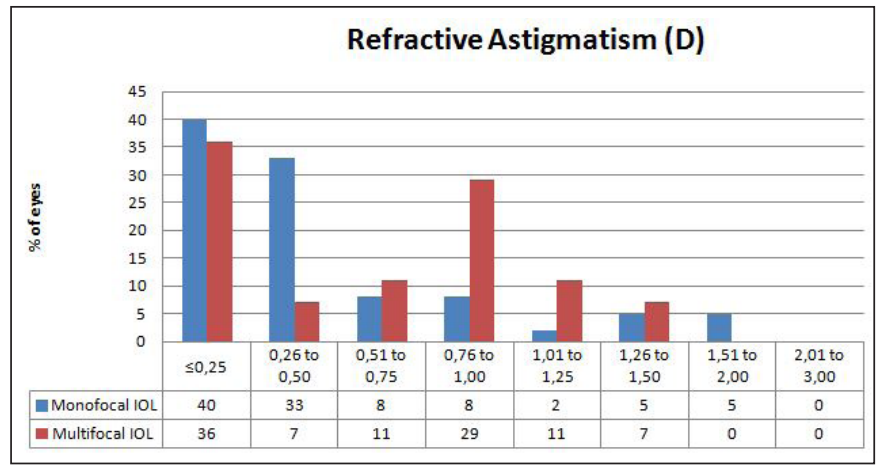

Figure B. Comparison of postoperative refractive astigmatism frequency at 30 days between monofocal toric intraocular lenses (IOLs) $(n=63)$ and multifocal toric IOLs $(n=28)$ for different powers.

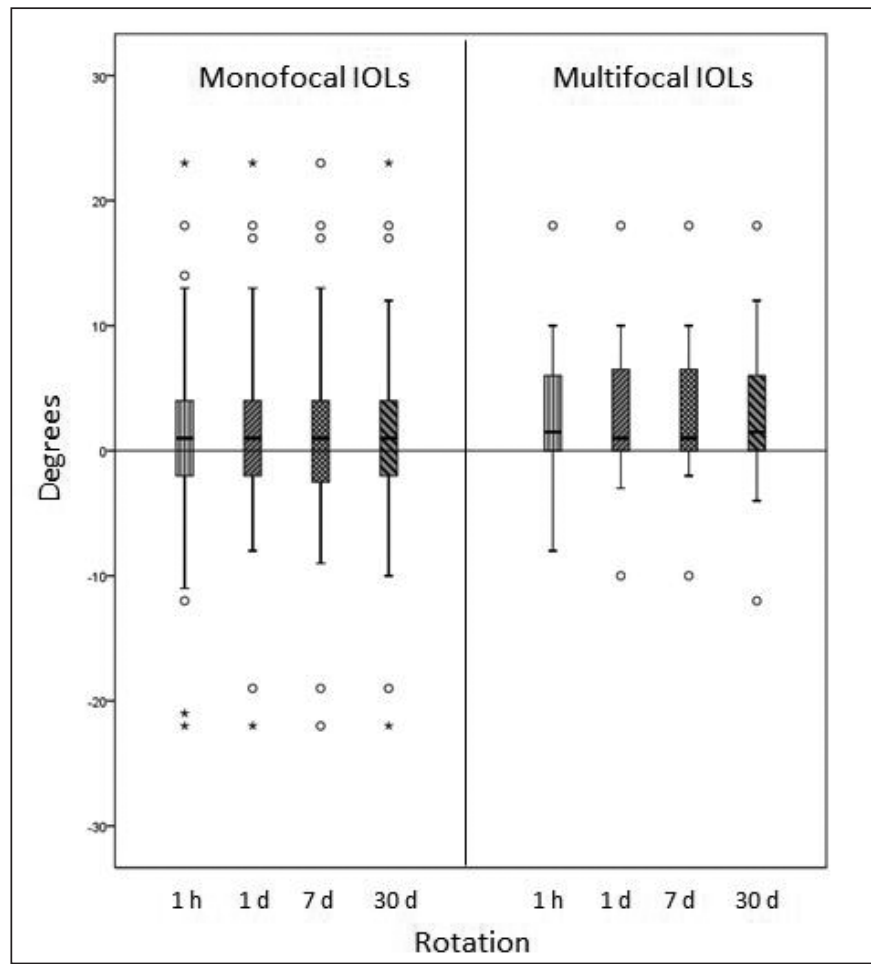

Figure C. Box and whiskers plot of rotation of monofocal toric intraocular lenses (IOLs) $(n=63)$ and multifocal toric IOLs $(n=28)$ at each followup visit. Values are represented as median and quartiles. 